\title{
Miscibility Studies of Hydroxypropyl Cellulose/Poly(Ethylene Glycol) in Dilute Solutions and Solid State
}

\author{
K. Sudharsan Reddy, ${ }^{1}$ M. N. Prabhakar, ${ }^{1}$ P. Kumara Babu, ${ }^{1}$ G. Venkatesulu, ${ }^{1}$ \\ U. Sajan Kumarji Rao, ${ }^{2}$ K. Chowdoji Rao, ${ }^{1}$ and M. C. S. Subha ${ }^{2}$ \\ ${ }^{1}$ Department of Polymer Science and Technology, Sri Krishnadevaraya University, Andhra Pradesh Anantapur 515003, India \\ ${ }^{2}$ Department of Chemistry, Sri Krishnadevaraya University, Andhra Pradesh Anantapur 515003, India
}

Correspondence should be addressed to K. Chowdoji Rao, chowdojirao@gmail.com

Received 31 May 2012; Revised 21 August 2012; Accepted 22 August 2012

Academic Editor: Yuriy A. Knirel

Copyright ( $\odot 2012$ K. Sudharsan Reddy et al. This is an open access article distributed under the Creative Commons Attribution License, which permits unrestricted use, distribution, and reproduction in any medium, provided the original work is properly cited.

\begin{abstract}
The miscibility of Hydroxypropyl cellulose (HPC)/poly(ethylene glycol) (PEG) blends over an extended range of concentrations in water. The viscosity, ultrasonic velocity, and refractive index of the above blend solutions have been measured at $30^{\circ} \mathrm{C}$. The interaction parameters such as $\Delta \beta$ and $\mu$ proposed by Chee and $\alpha$ proposed by Sun have been obtained using the viscosity data to probe the miscibility of the polymer blends. The values indicated that the blends were miscible when HPC content is more than $40 \%$ in the blend. The obtained results have been confirmed by the ultrasonic velocity and refractive index studies. The films of the blends were prepared by solution casting method using water as a solvent. The prepared films have been characterized by analytical techniques such as FTIR, DSC, X-RD, and SEM to probe the miscibility of HPC/PEG blends. The compatibility in the above compositions may be due to the formation of H-bonding between hydroxyl groups of HPC and etheric oxygen atom of PEG molecules.
\end{abstract}

\section{Introduction}

Usage of blended polymers is the most effective way to produce new multipurpose materials. During the past few years, researchers have paid considerable attention to the study of polymer blending [1-3].

A combination of synthetic and natural polymers results in new materials, which have specific properties of synthetic components (good mechanical properties, easy possibilities, low production, and transformation costs) and biocompatibility nature of biopolymers $[4,5]$. These blends have already been utilized as biodegradable biomaterials [6], drug delivery systems [7], membranes [8], materials for agricultural applications [9], and so forth.

Blending of polymers is one of the simplest methods to obtain a variety of physical and chemical properties from the constituent polymers at a molecular level. The gain in newer properties depends on the degree of compatibility or miscibility of the polymers. There have been number of techniques to study the miscibility of polymer blends such as FTIR, DSC,
X-RD, and SEM. Most of these techniques may be complicated, costly, and time consuming. Chee [10] and Sun et al. [11] suggested viscometry for the study of the polymerpolymer miscibility. Palladhi and Singh [12, 13] showed that the variation of ultrasonic velocity and viscosity with blend composition is linear for miscible and nonlinear for immiscible blends.

Hydroxypropyl cellulose (HPC) is a cellulose derivative employed as coatings, excipients, encapsulations, binding materials, foaming agents, protection colloids, flocculants, and so forth, for a wide variety of applications in food, drugs, paper, ceramics, plastics, and so forth [14]. Hydroxypropyl cellulose films are used as a food additive, HPC is used as a thickener, a low level binder and as an emulsion stabiliser with E number E463. In pharmaceuticals it is used as a disintegrant, and a binder, in tablets [15]. They act as acceptable barriers to moisture and oxygen $[16,17]$. Blends based on HPC with natural or synthetic polymers were proposed to design new materials with enhanced properties and with wide range of applications. 
Poly(ethylene glycol) is used in all applications of aqueous phase partitioning. PEG is a biocompatible and biodegradable polymer and has adhesive and flocculent properties [18]. For biochemical separations on the laboratory scale, the most commonly used aqueous phase system is composed of Dextron and PEG [19].

In the present study HPC and PEG polymer blend solutions were prepared in water and their miscibility was studied by using viscosity, ultrasonic velocity, and refractive index measurements. By solution casting method these polymer blend films were also prepared and their miscibility studied using FTIR, DSC, X-RD, and SEM techniques. The compatibility of these polymer blends is discussed and it is concluded that the presence of hydrogen bonding groups $[20,21]$ in these two polymers is responsible for the polymerpolymer interactions [22]. These results are presented here.

\section{Materials and Experimental Techniques}

2.1. Materials. Hydroxypropyl cellulose $\left(M_{W}=1,40,000\right)$ was purchased from Sigma Aldrich, USA, poly(ethylene glycol) $\left(M_{W}=6,000\right)$ was purchased from Qualigens, Mumbai, (India) and both were used without further purification. Double distilled water having zero conductivity (distilledwater usually runs between 2 and $10 \mathrm{~S} / \mathrm{cm}$ ) was used as a solvent.

2.2. Preparation of Blend Solutions. Two kinds of polymer powders (HPC and PEG) were dried in a vacuum oven for $24 \mathrm{hrs}$ at $50^{\circ} \mathrm{C}$ to remove the free water from the samples before use. HPC and PEG were separately dissolved in water to form $1 \%(\mathrm{w} / \mathrm{v})$ solution. It is worth mentioning that in the preparation of HPC solution, the HPC powder must be slowly added to the solvent with gently stirring for about one week at room temperature to obtain a homogenous solution; otherwise HPC tends to form sticky clumps when large amounts of samples were added to the solvent. The blends of stock solutions $(20 / 80,40 / 60,50 / 50,60 / 40$, and 80/20) of HPC/PEG were prepared by stirring the mixtures at room temperature for about 45 minutes. Using the above pure and blended stock solutions different blend solutions $(0.1,0.3$, $0.5,0.7$, and $0.9(\mathrm{w} / \mathrm{v})$ concentrations) were prepared.

2.3. Preparation of Blend Films. Thin films of the polymers and their blends were prepared by solution casting method. Separate aqueous solutions of HPC and PEG were prepared. A solution of HPC was added to that of PEG with constant stirring. The mixtures were stirred for $45 \mathrm{~min}$ at room temperature to ensure complete mixing. The total polymer concentration was kept at $1 \%(\mathrm{w} / \mathrm{v})$. Stock solutions of HPC and PEG and their different blend compositions were then casted onto a Teflon-coated clean glass plate and dried using IR lamp in a dust-free atmosphere. The dried thin films were peeled off from the glass plate and were found to be transparent. The prepared thin films of pure polymers and blends were characterized using FTIR, DSC, X-RD, and SEM methods.
2.4. Techniques. Viscosity and density measurements were made at $30^{\circ} \mathrm{C}$ using Ubbelohde suspended level viscometer (with the flow time of $95 \mathrm{sec}$ for distilled water) and specific gravity bottle, respectively. The required temperature $\left(30^{\circ} \mathrm{C}\right)$ was maintained within $\pm 0.05^{\circ} \mathrm{C}$. The ultrasonic velocities of the blend solutions with different compositions, namely, $0 / 100,20 / 80,40 / 60,50 / 50,60 / 40,80 / 20$, and $100 / 0$ by weight, were measured at $30^{\circ} \mathrm{C}$ using ultrasonic interferometer. The constant temperature was maintained by circulating water from a thermostat with a thermal stability of $\pm 0.05^{\circ} \mathrm{C}$ through the double-walled jacket of ultrasonic experimental cell. The experimental frequency was $2 \mathrm{MHz}$, and the velocity measurements were accurate to better than $\pm 0.5 \%$. The refractive indices of blend solutions with different compositions were measured directly with an Abbe refractometer (digital) with thermostated water circulation system at $30^{\circ} \mathrm{C}$. The accuracy of the refractive index measurement is $\pm 0.02 \%$.

2.5. Fourier Transform Infrared Spectroscopy. Fourier transform infrared (FTIR) spectra of HPC, PVP, and their blend films were taken using Bomen MB-3000 FTIR spectrometer. Blend films were characterized at room temperature from 4000 to $400 \mathrm{~cm}^{-1}$ under an $\mathrm{N}_{2}$ atmosphere at a scan rate of $21 \mathrm{~cm}^{-1}$.

2.6. Differential Scanning Calorimetry. DSC curves of HPC, PVP, and their blend films of different compositions were recorded using TA instruments Differential Scanning Calorimeter (Model: SDT Q600, USA). The analysis of samples was performed at heating rate of $20^{\circ} \mathrm{C} / \mathrm{min}$ under $\mathrm{N}_{2}$ atmosphere at a purge speed of $100 \mathrm{~mL} / \mathrm{min}$.

2.7. X-Ray Diffraction. The X-ray Diffraction (XRD) patterns of the blend samples were obtained with an Intel diffractometer (Paris, France) with monochromatized $\mathrm{Cu} \mathrm{K} \alpha$ radiation ( $\operatorname{scan}$ speed of $1^{\circ} / \mathrm{min}$ in a $2 \theta$ range of $5^{\circ}-40^{\circ}$ ) at room temperature.

\section{Results and Discussion}

3.1. Viscosity Studies. The absolute viscosity versus concentration curves for the blends of HPC and PEG of different compositions at $30^{\circ} \mathrm{C}$ in water is shown in Figure 1. It was well established earlier by many workers $[23,24]$ that the variations of viscosity versus concentration of blend composition plots are linear for compatible blends and nonlinear for incompatible blends. On this basis in the present study it is noticed that absolute viscosity varied nonlinearly with the concentration of blend composition up to 40/60 HPC/PEG and linearly beyond this composition. This indicates that the polymer blends of HPC/PEG are found to be immiscible when HPC content is up to $40 \%$ and then miscible beyond this composition.

From Figure 1, it is also observed that as the concentration of HPC increases in the blend composition, the absolute viscosity also increases. With the increasing percentage of HPC in the HPC/PEG blend, PEG can interact with several chains of HPC and can form highly cross-linked structure, 


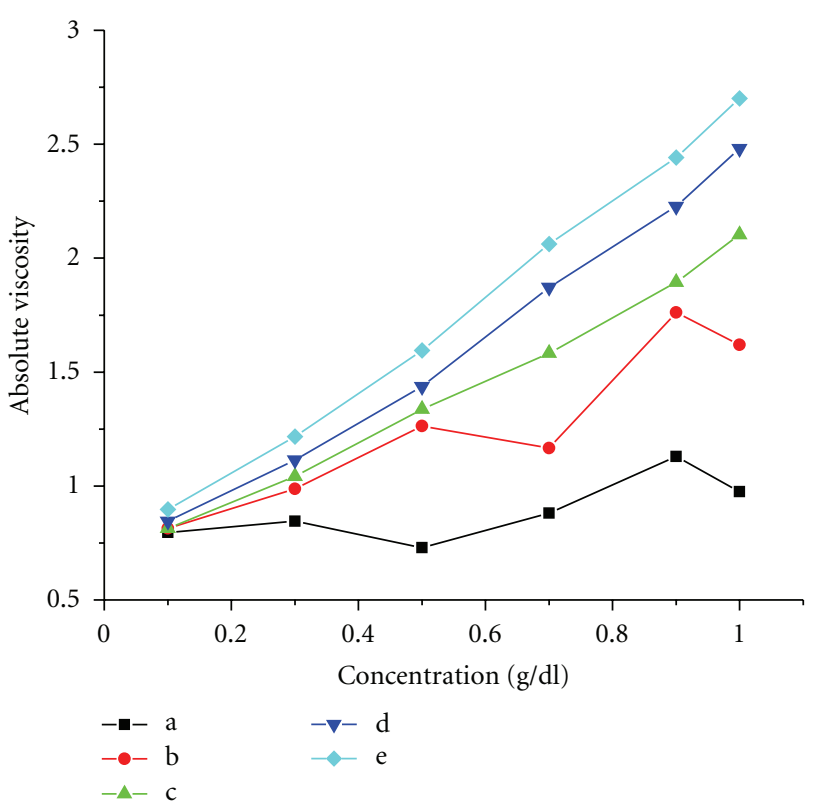

Figure 1: Plots of absolute viscosity versus concentration for $1 \%$ w/v HPC-PEG blends of 20/80 (a), 40/60 (b), 50/50 (c), 60/40 (d), $80 / 20$ (e).

which causes high solution viscosity. Further, with the increase in proportion of HPC in the blend along with crosslinking effect, the effect of hydrogen bonding between the $\mathrm{OH}$ groups of HPC and etheric oxygen atom of PEG also increases. These effects at higher contents of HPC of these blends lead to the high viscosity of the blends as observed in Figure 1.

The Huggins plots of reduced viscosity against concentration of different compositions of $1 \%$ (w/v) HPC/PEG blends pure HPC and pure PEG in water at $30^{\circ} \mathrm{C}$ are shown in Figures 2(a)-2(g). From these graphs on extrapolating to zero concentration, the intrinsic viscosities are determined, and the values of HPC, PEG, and HPC/PEG blends of different compositions $(20 / 80,40 / 60,50 / 50,60 / 40,80 / 20)$ are $1.3258,0.1373,0.0426,0.1956,0.555,0.5184$, and $0.7529 \mathrm{dL} / \mathrm{g}$, respectively. The intrinsic viscosity of HPC/PEG blends for different compositions is in between the values of pure polymers. The reasonable interpretation may be that the attractive interactions are existing between HPC and PEG in water. These attractive interactions may lead to decrease the intermolecular excluded volume effect and decrease the hydrodynamic volume. As a consequence, the HPC/PEG blend coils expand, which causes an increase of the intrinsic viscosity of HPC/PEG blends in water.

From these graphs (Figures 2(a)-2(e)), it is also clearly evident that the Huggins curves composed of two regions with varying slopes for the polymer blends up to $40 / 60$ HPC/PEG blend composition. The change in the slope of the curve may be attributed to the mutual repulsion of macromolecules in solution which favors the polymer immiscibility up to $40 \%$ of HPC content in the blend. But later, that is, above $40 \%$ HPC content in the blend composition, the reduced viscosity versus concentration of

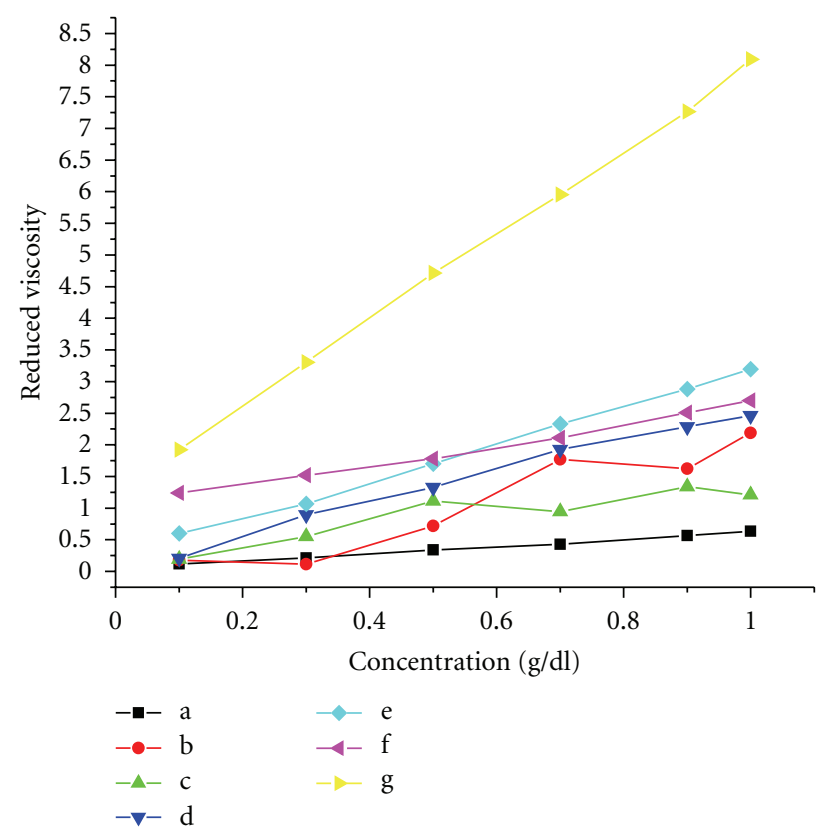

FIgure 2: Reduced viscosity versus concentration curves for pure PEG (a), HPC/PEG blends 20/80 (b), 40/60 (c), 50/50 (d), 60/40 (e) $80 / 20$ (f) and pure HPC (g).

TABle 1: Chee and Sun's interaction parameters for different composition of $1 \%(\mathrm{w} / \mathrm{v}) \mathrm{HPC} / \mathrm{PEG}$ blends in water at $30^{\circ} \mathrm{C}$.

\begin{tabular}{|c|c|c|c|}
\hline \multirow{2}{*}{$\begin{array}{l}\text { HPC/PEG } \\
\text { blend } \\
\text { composition }\end{array}$} & \multicolumn{2}{|c|}{$\begin{array}{l}\text { Chee's differential interaction } \\
\text { parameters }\end{array}$} & \multirow{2}{*}{$\begin{array}{l}\text { Sun's miscibility } \\
\text { parameter } \\
\alpha \times 10^{2} \\
\end{array}$} \\
\hline & $\Delta B$ & $\mu$ & \\
\hline $20 / 80$ & -0.0821 & -1.1578 & -2.4609 \\
\hline $40 / 60$ & -0.1609 & -0.3186 & -0.3344 \\
\hline $50 / 50$ & 0.1238 & 8.1672 & 7.7292 \\
\hline $60 / 40$ & 0.0504 & 0.5192 & 0.054 \\
\hline $80 / 20$ & 0.0184 & 0.9732 & 0.0252 \\
\hline
\end{tabular}

blends shows linear variation which is an indication of mutual attraction of molecules in solution which favors the polymer miscibility. A similar observation was made by Vasanthakumari et al. [25] from their viscosity studies on miscibility of poly(2-hydroxyethylmethocrylate) and hydroxyl propyl methyl cellulose blends.

3.2. Chee and Sun Interaction Parameters. In order to quantify the miscibility or immiscibility nature of the present polymer blend studies, the interaction parameters $\Delta B$ and $\mu$ suggested by Chee and Sun's interaction parameter $\alpha$ are calculated with the help of (1), (4), and (5) for the HPC/PEG blend systems in water at $30^{\circ} \mathrm{C}$ and are presented in the Table 1.

Chee suggested that the general expression for interaction parameter when polymers are mixed in weight fractions $w_{1}$ and $w_{2}$ is as follows:

$$
\Delta B=\frac{b-\bar{b}}{2 w_{1} w_{2}},
$$


where $\bar{b}=w_{1} b_{11}+w_{2} b_{22}$ in which $b_{11}$ and $b_{12}$ are the slopes of the viscosity curves for the pure components. The coefficient $b$ is related to the Huggins coefficient $K_{\mathrm{H}}$ as

$$
b=K_{\mathrm{H}}[\eta]^{2} .
$$

For ternary systems, the coefficient $b$ is also given by

$$
b=w_{1}^{2} b_{11}+w_{2}^{2} b_{22}+2 w_{1} w_{2} b_{12},
$$

where $b_{12}$ is the slope for the blend solution. Using these values, Chee defined a more effective parameter as follows:

$$
\mu=\frac{\Delta B}{\left\{[\eta]_{2}-[\eta]_{1}\right\}^{2}}
$$

where $\eta_{1}$ and $\eta_{2}$ are the intrinsic viscosities for the pure component solutions. Recently, Sun et al. has suggested a new formula for the determination of polymer miscibility as follows:

$$
\alpha=K_{m}-\frac{K_{1}[\eta]_{1}^{2} w_{1}^{2}+K_{2}[\eta]_{2}^{2} w_{2}^{2}+2 \sqrt{K_{1} K_{2}}\left[\eta_{1}\right]\left[\eta_{2}\right] w_{1} w_{2}}{\left\{[h]_{1} w_{1}+[h]_{2} w_{2}\right\}^{2}},
$$

where $K_{1}, K_{2}$ and $K_{m}$ are the Huggins constants for individual components 1, 2 and the blend, respectively.

It is observed from Table 1 that the values of $\Delta B$, $\mu$, and $\alpha$ are negative up to $40 / 60 \mathrm{HPC} / \mathrm{PEG}$ blend composition and positive beyond this composition.

In general $[23,24]$ if $\Delta B, \mu$, and $\alpha$ are positive for any polyblend system, these are considered to be as miscible one whereas if these values are negative, the polyblends are considered as immiscible one. Based on this it is concluded from the present study, as per the values given in Table 1, that HPC/PEG blend shows immiscibility when HPC content is up to $40 \%$ and then miscible beyond this composition. The probable reason for this may be that up to $40 / 60 \mathrm{HPC} / \mathrm{PEG}$ blends, that is, in the PEG rich content blend composition the etheric "O" atoms are responsible for H-bonding with -OH groups of HPC (Scheme 1).

$\mathrm{H}$-bonding between HPC and PEG can be explained as follows. The available $\mathrm{OH}$-groups of HPC are less in number up to 40/60 HPC/PEG blend; hence the hydrogen bonding possibility is less, and this may be the reason for immiscibility of these blend compositions, that is, up to $40 \%$ HPC content. Later as the HPC content increases beyond this composition more no. of HPC molecules are available and are responsible for having more no. of $-\mathrm{OH}$ groups which in turn gives a scope to form more H-bonding with etheric oxygen of PEG molecules. This leads to miscibility of this blend beyond $40 \%$ HPC $+60 \%$ PEG. A similar observation was reported by Ravi Prakash and Rai [26] from their work on the miscibility studies of Sodium Alginate/PEG blends in water by viscosity, ultrasonic velocity, and refractive index methods.

3.3. Ultrasonic Velocity Studies. The variation of ultrasonic velocity $(v)$ with the concentration of different compositions

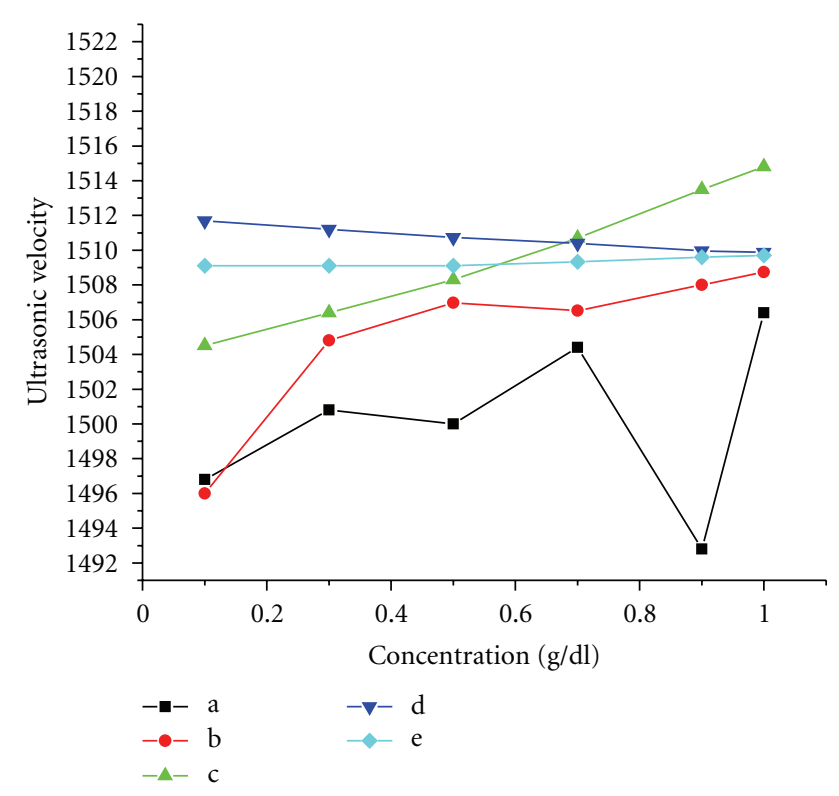

FIGURE 3: Ultrasonic velocity versus concentration curves for HPC/PEG blends of 20/80 (a), 40/60 (b), 50/50 (c), 60/40 (d), 80/20 (e).

of $1 \%(\mathrm{w} / \mathrm{v})$ of $\mathrm{HPC} / \mathrm{PEG}$ blends in water is plotted in Figures 3(a)-3(e).

It has been observed from Figures 3(a) to 3(e) that ultrasonic velocity varies nonlinearly up to $40 / 60$ (HPC/PEG) blend composition and linearly with polymer blend concentration beyond this blend composition. It was well established [27-29] that for a miscible blend, the ultrasonic velocity varies linearly with polymer blend concentration, and for an immiscible blend it varies nonlinearly with polymer blend concentration. This indicates HPC and PEG form immiscible blend in water up to $40 / 60$ (HPC/PEG) blend composition and miscible beyond this composition.

From these results, we can conclude that the variation of the curves relating the different acoustical parameters indicates immiscibility nature of (HPC/PEG) blend up to $40 / 60 \mathrm{HPC} / \mathrm{PEG}$ blend composition, and beyond this composition these blends behave as miscible. A similar observation was reported by Illiger et al. [24] from their ultrasonic investigation on compatibility of polymer blends.

3.4. Refractive Index Studies. It is evident from Figures 4(a)$4(\mathrm{e})$ that the refractive index varies non-linearly with the concentration up to 40/60 HPC/PEG blend composition, whereas it varies linearly beyond this composition. This behavior indicates that HPC/PEG blends are immiscible up to $40 / 60$ blend composition, and beyond this composition these blends are miscible. A similar observation was reported by Basavaraju et al. in [30] case of Xanthan gum/PEO blends.

These conclusions are in good agreement with the results obtained for this blend system from viscometric and ultrasonic studies. 


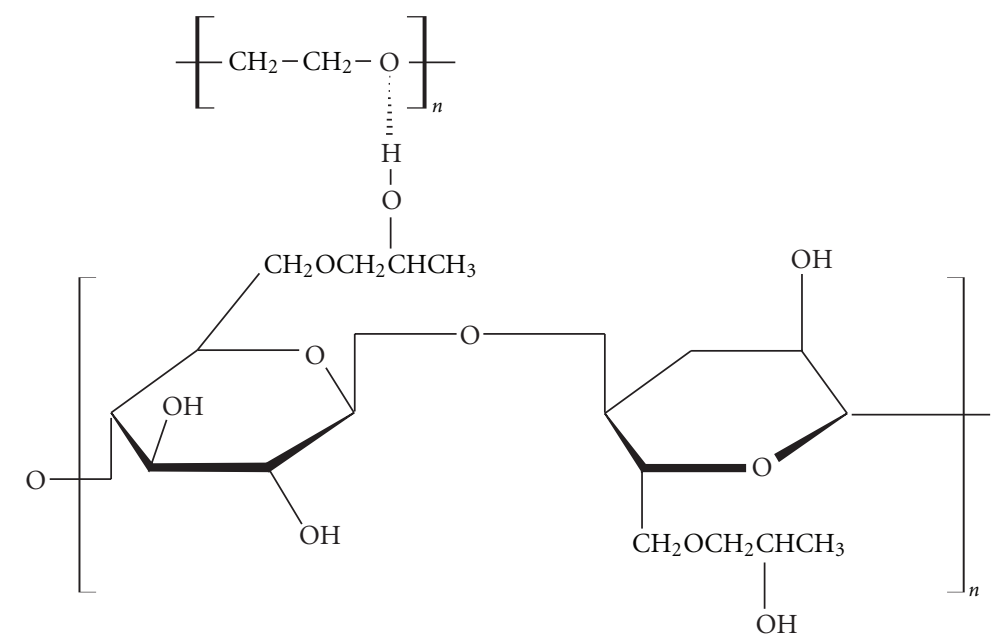

Scheme 1: Hydrogen bonding between the Hydroxyl groups of PEG and the hydroxyl groups of HPC.

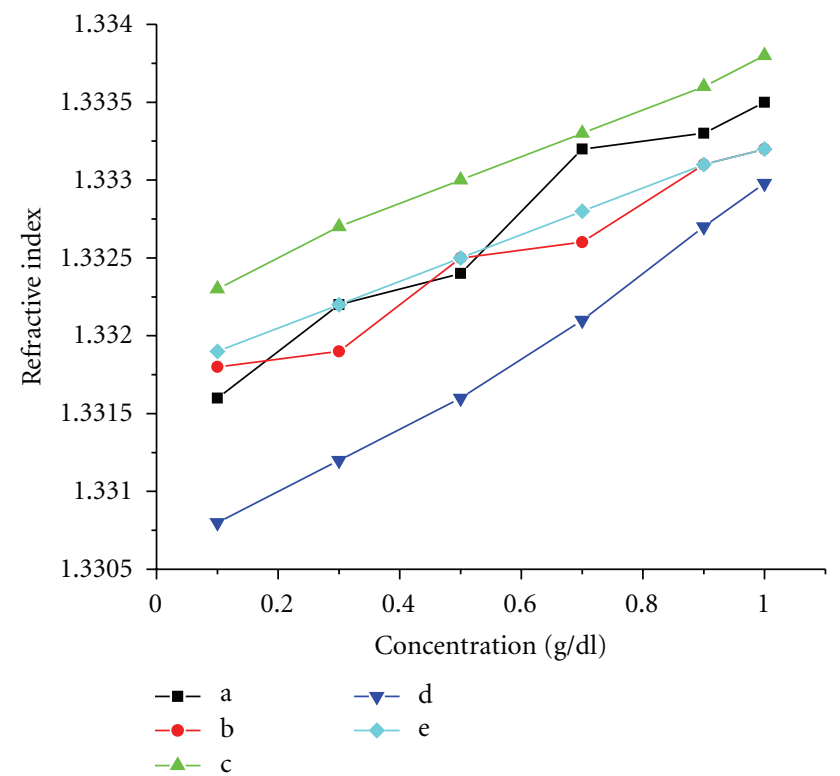

Figure 4: Refractive Index versus concentration curves for HPC/PEG blends of 20/80 (a), 40/60 (b), 50/50 (c), 60/40 (d), 80/20 (e).

\section{Miscibility Studies of Blend Films by Characteristic Techniques}

4.1. Fourier Transform Infrared Spectroscopy Studies. FTIR spectroscopy has been widely used by many researchers to study the formation of blends [31-33]. FTIR spectrum provides information regarding intermolecular interactions corresponding to stretching or blending vibrations of particular bands, and the positions at which these peaks appear depend directly on the force constant or band strength. Hydrogen bonding or other secondary interactions between chemical groups on the dissimilar polymers should theoretically cause a shift in peak position of the participating groups. This kind of behavior is exhibited by miscible blends that show

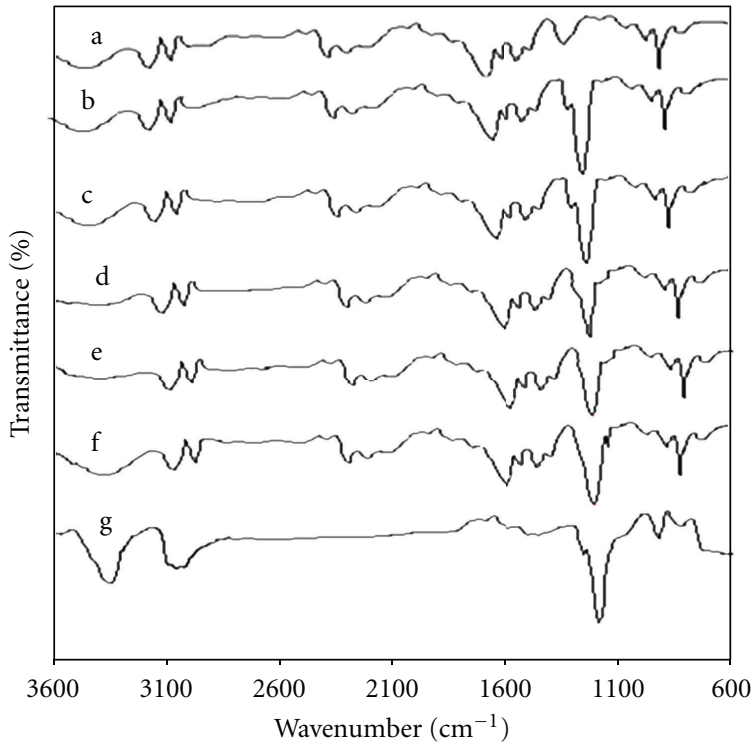

Figure 5: FTIR spectra of PEG (a), 20/80 HPC/PEG (b), 40/60 HPC/PEG (c), 50/50 HPC/PEG (d), 60/40 HPC/PEG (e), 80/20 HPC/PEG (f), HPC (g).

extensive phase mixing. Hydrogen bonding interactions usually move the stretching frequencies of participating groups, for example, $\mathrm{O}-\mathrm{H}$ towards lower numbers usually with increased intensity and peak broadening. The shift in peak position will depend on the strength of the interaction.

The FTIR spectra of HPC, PEG, and the blends of HPC/PEG (20/80, 40/60, 50/50, 60/40 and 80/20) are shown in Figure 5. The formation of strong hydrogen bonds between HPC and PEG was demonstrated by FTIR spectroscopy from the shifts of absorption bands showing hydroxyl stretching vibrations, which were sensitive to the hydrogen bonds formed during blending. The broad transmission bands at $3600-3100 \mathrm{~cm}^{-1}$ produced by stretching of the hydroxyl groups in the spectrum of HPC can be 


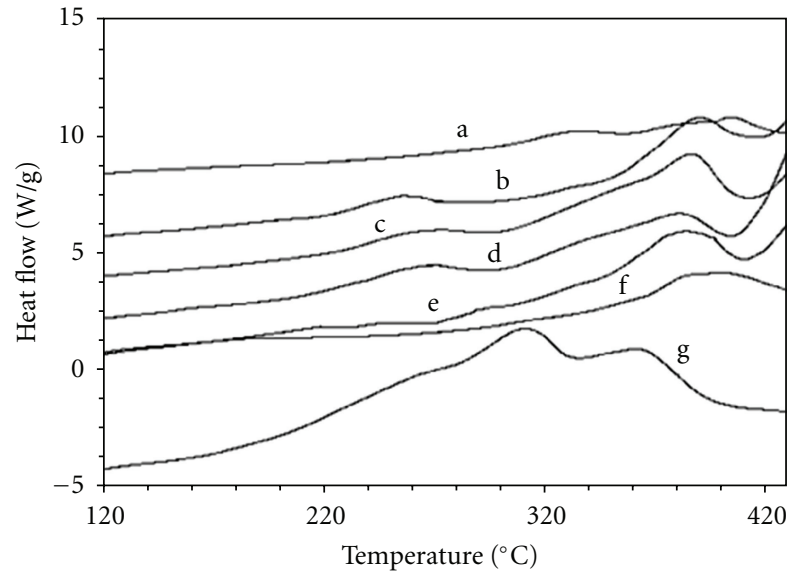

Figure 6: DSC thermograms of HPC (a), HPC/PEG 80/20(b), HPC/PEG 60/40 (c), HPC/PEG 50/50 (d), HPC/PEG 40/60 (e), HPC/PEG 20/80 (f), PEG (g).

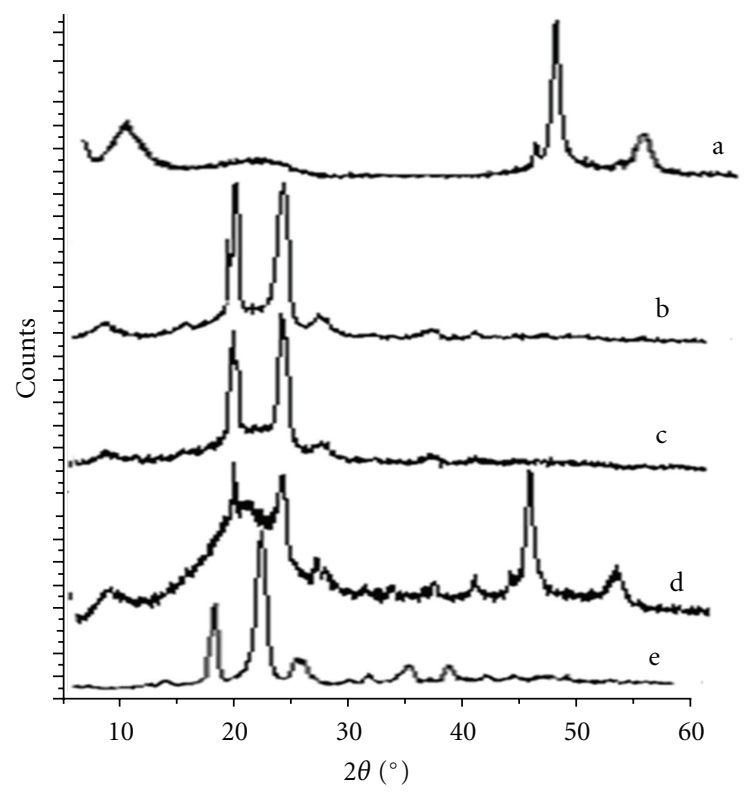

Figure 7: X-ray diffraction patterns of HPC (a), HPC/PEG 80/20 (b), HPC/PEG 50/50 (c), HPC/PEG 20/80 (d), PEG (e).

remarkably distinguished. It can be seen from the spectra that the peak intensity and the peak shape were clearly different, and these differences were induced by the different blend ratios. The presence of hydrogen-bond structures in some blends could be inferred from the peak shape and peak intensity of the absorption band of the hydroxyl starching vibrations in the FTIR spectra [34]. The broadband in the pure HPC spectrum at $3600-3100 \mathrm{~cm}^{-1}$, with a maximum at $3460 \mathrm{~cm}^{-1}$, was assigned to stretching vibrations of the $-\mathrm{OH}$ groups. The difference among the curves in Figure 5, a little broadening or shifting, or a peak at $3600-3100 \mathrm{~cm}^{-1}$ was observed in the transmission band of the HPC/PEG blends when they were compared with that of pure HPC, which suggested that a relative low amount of interaction was presented between the polymers.

From Figures 5(b) and 5(c) it is clear that 20/80, 40/60 HPC/PEG blends, there is no change (or) shift in -OH stretching vibrations. In these blends as the HPC content is lower (40\%) and no. of $\mathrm{OH}$ groups available also less to form the hydrogen bonds with etheric oxygen of PEG molecules. Hence these blends show immiscible nature. By observing (d, $\mathrm{e}, \mathrm{f}$, and g) spectrum it is clear that the hydroxyl stretching vibrations shifted to a lower wave number with increasing amounts of HPC which are responsible for formation of more hydrogen bonding interactions between the $\mathrm{OH}$ groups belonging to HPC and etheric oxygen belonging to PEG molecules. This leads to miscibility nature of HPC/PEG blends, where the HPC content is more than $40 \%$ in the blend compositions.

4.2. Differential Scanning Calorimetry Studies. Figure 6 shows DSC thermogram of HPC, PEG, and their different blend compositions (20/80, 40/60, 50/50,60/40, and 80/20). DSC thermograms were carried out to determine the compatibility of the polymer blend. $T_{m}$ values for pure HPC and Pure PEG are about $353^{\circ} \mathrm{C}$ and $333^{\circ} \mathrm{C}$, respectively. As shown in Figure 6 broad endothermic peaks, observed in case of 50/50, 60/40, and 80/20 HPC/PEG blend films, which indicates hydrogen bonding interactions between HPC and PEG. By careful observation in blends where HPC content is less than $40 \%$, that is, in case of DSC thermograms of $20 / 80$ and 40/60 HPC/PEG blends the narrow peaks are noticed which shows the non-H-bonding nature between HPC and PEG. From this we can conclude that for blend where the content of HPC is more than $40 \%$ they are miscible whereas the blends were the content of HPC is less than $40 \%$ they are immiscible.

4.3. X-Ray Diffraction Studies. The measurement of the X$\mathrm{RD}$ patters of polymer blend is also used as a criterion to determine its miscibility. If the two components have low compatibility, then each polymer would have its own crystal region in the blend films, and X-ray patterns of the sample would express as simply mixed patterns with the same ratio as those for blending. The typical X-RD patterns of PEG, HPC, and their blend compositions are shown in Figure 7. For the pure HPC (a), there were two peaks around $2 \theta=8.5^{\circ}$ and $44.6^{\circ}$. The diffraction model of PEG (b) showed at $2 \theta=19.7$ and 23.9. Peak of 50/50 (c), HPC/PEG blend film is $2 \theta=19.9^{\circ}$ and $23.9^{\circ} ; 80 / 20$ $\mathrm{HPC} / \mathrm{PEG}$ (d) blend film is $2 \theta=19.5^{\circ}$ and $23.7^{\circ}$. These peaks are similar to PEG. These evidences further concluded that strong interaction occurred between HPC/PEG molecules in the blends of 50/50 HPC/PEG and 80/20 HPC/PEG which is an indication of miscibility nature. But in case of 20/80 blend film showed $2 \theta=19.4$ and 44.6 which indicates that there is no interaction between HPC and PEG molecules in the 20/80 blend film; each component has shown its own crystal region in the blend film, and X-RD patterns have expressed simple mixed patterns. These evidences further concluded 


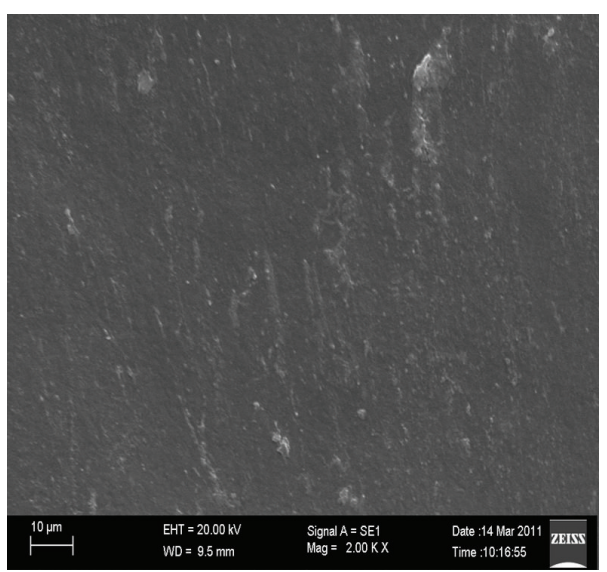

(a)

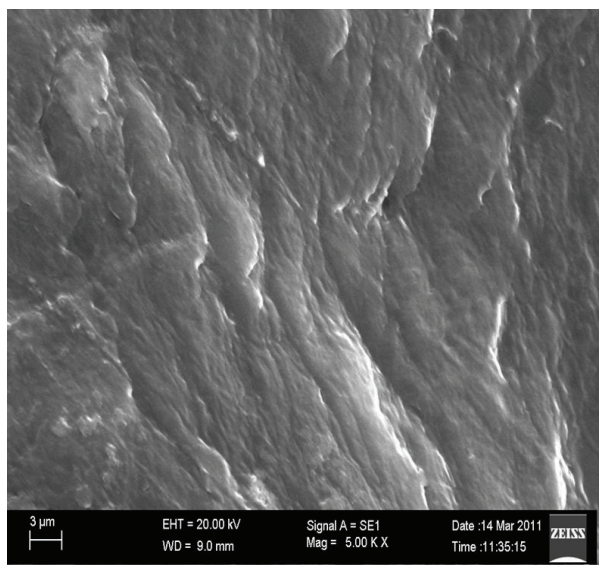

(c)

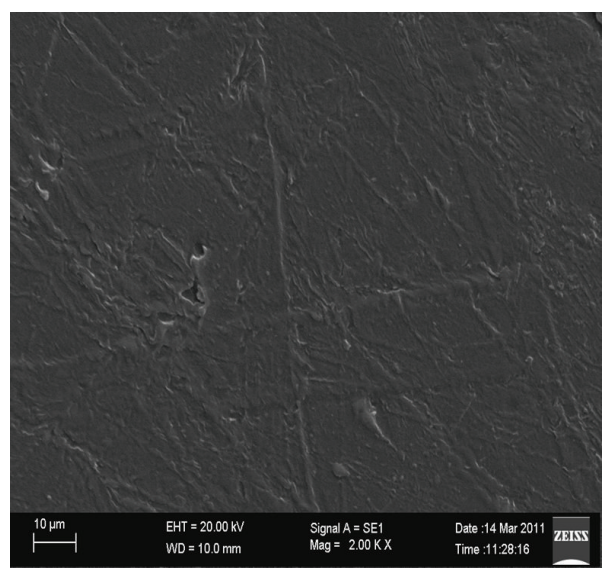

(b)

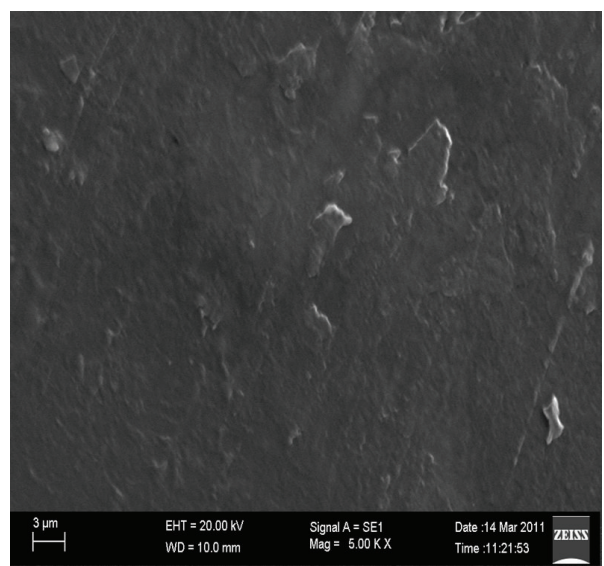

(d)

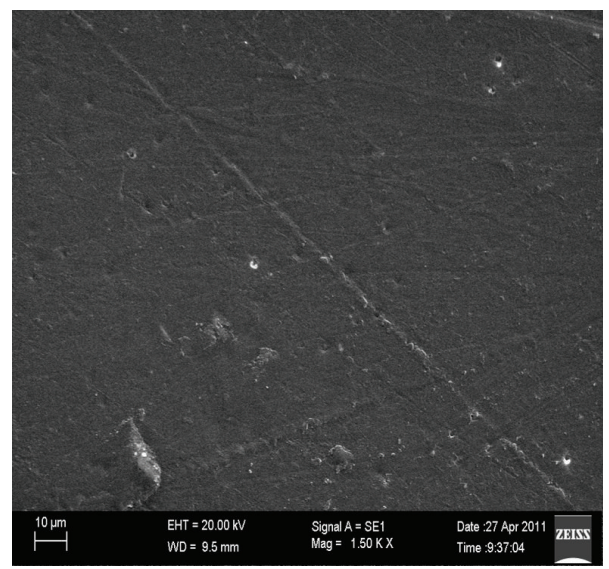

(e)

Figure 8: SEM images of HPC (a), PEG (b), 20/80 HPC/PEG (c), 50/50 HPC/PEG (d), 80/20 HPC/PEG (e).

that 20/80 HPC/PEG is immiscible, and 50.50, 80/20 blend compositions are miscible.

4.4. Scanning Electron Microscopy. Figure 8 shows the SEM images of HPC (a), PEG (b), and their blends of different compositions 20/80 (c), 50/50 (d), and 80/20 (e). The surface morphology of HPC (a) and PEG (b) films was homogeneous. The bright strips presented in the image of pure PEG. With the addition of PEG to HPC, the morphologies of the blended films changed dramatically. There is no obvious phase separation observed in the 50/50 and $80 / 20 \mathrm{HPC} / \mathrm{PEG}$ blend films, but phase separation is visible in the 20/80 HPC/PEG blend. Hence, we conclude that the blends of 50/50, $80 / 20$ are miscible, and 20/80 of 
$\mathrm{HPC} / \mathrm{PEG}$ blend is immiscible in nature. This confirms the HPC/PEG blends where the HPC content is more $40 \%$ are miscible and the blends where HPC content is less $40 \%$ are immiscible. This further supports the conclusions drawn from other analytical techniques in case of the miscibility nature of this blend.

\section{Conclusions}

The miscibility of HPC/PEG blends in water has been studied by solution methods such as viscosity, ultrasonic velocity, and refractive index techniques at $30^{\circ} \mathrm{C}$. Using the viscosity data, interaction parameters $\Delta B, \mu$, and $\alpha$ are were computed. These values revolved that the blend is miscible when HPC content is more than $40 \%$ in the blend. This was further confirmed by ultrasonic velocity and refractive index measurements. Below this critical HPC concentration, the blends were found to be immiscible.

The prepared films of the HPC/PEG blends are also characterized for FTIR, DSC, X-RD, and SEM to support the data obtained from simple methods such refractive index, ultrasonic velocity, and viscosity.

\section{References}

[1] M. Barsbay and A. Güner, "Miscibility of dextran and poly(ethylene glycol) in solid state: effect of the solvent choice," Carbohydrate Polymers, vol. 69, no. 2, pp. 214-223, 2007.

[2] G. S. Guru, P. Prasad, H. R. Shivakumar, and S. K. Rai, "Miscibility studies of polysaccharide xanthan Gum/PVP blend," Journal of Polymers and the Environment, vol. 18, no. 2, pp. 135-140, 2010.

[3] T. M. M. Swamy, B. Ramaraj, and Siddaramaiah, "Thermal and morphological properties of SA/HPMC blends," Journal of Applied Polymer Science, vol. 112, no. 4, pp. 2235-2240, 2009.

[4] Y. Nishio, N. Hirose, and T. Takahashi, "Miscibility studies of hydroxy propyl cellulose/ poly (ethylene glycol) in dilute solutions and solid state," Sen' Gakkashi, vol. 46, p. 441, 1990.

[5] Y. Nishio and R. S. J. Manley, "Blends of cellulose with nylon 6 and poly( $\varepsilon$-caprolactone) prepared by a solution-coagulation method," Polymer Engineering \& Science, vol. 30, no. 2, pp. 7182, 1990.

[6] Y. Kikuchi, N. Kubota, H. Fukuma, and H. Mitsuishi, "Preparation and properties of macromolecular complexes consisting of chitosan derivatives, iron(III) hydroxide sulfate, and poly(potassium vinyl sulfate)," Macromolecular Rapid Communications, vol. 17, no. 2, pp. 131-136, 1996.

[7] N. Kubota and Y. Kikuchi, "Preparation and properties of macromolecular complexes consisting of chitosan derivatives and potassium metaphosphate," Molecular Chemistry and Physics, vol. 193, no. 2, pp. 559-566, 1992.

[8] N. Kubota and Y. Eguchi, "Facile preparation of water-soluble $\mathrm{N}$-acetylated chitosan and molecular weight dependence of its water-solubility," Polymer Journal, vol. 29, no. 2, pp. 123-127, 1997.

[9] N. Kubota, G. Konaka, and Y. Eguchi, "Characterization of blend films of chitin regenerated from porous chitosan with poly (vinyl alcohol)," Sen'i Gakkaishi, vol. 54, no. 4, pp. 212218, 1998.
[10] K. K. Chee, "Determination of polymer-polymer miscibility by viscometry," European Polymer Journal, vol. 26, no. 4, pp. 423-426, 1990.

[11] Z. Sun, W. Wang, and Z. Feng, "Criterion of polymer-polymer miscibility determined by viscometry," European Polymer Journal, vol. 28, no. 10, pp. 1259-1261, 1992.

[12] R. Paladhi and R. P. Singh, "Miscibility and interaction studies on some aqueous polymer blend solutions by ultrasonic and rheological techniques," Journal of Applied Polymer Science, vol. 51, no. 9, pp. 1559-1565, 1994.

[13] R. Paladhi and R. P. Singh, "Ultrasonic and rheological investigations on interacting blend solutions of poly(acrylic acid) with poly(vinyl pyrrolidone) or poly(vinyl alcohol)," European Polymer Journal, vol. 30, no. 2, pp. 251-257, 1994.

[14] M. G. Cascone, L. Di Silvio, B. Sim, and S. Downes, "Collagen and hyaluronic acid based polymeric blends as drug delivery systems for the release of physiological concentrations of growth hormone," Journal of Materials Science, vol. 5, no. 910, pp. 770-774, 1994.

[15] Myra L. Weiner and Lois A. Kotkoskie, Excipient Toxicity and Safety, 1999.

[16] R. D. Hagenmaier and P. E. Shaw, "Moisture permeability of edible films made with fatty acid and (hydroxypropyl)methylcellulose," Journal of Agricultural and Food Chemistry, vol. 38, no. 9, pp. 1799-1803, 1990.

[17] J. F. Hanlon, Handbook of Package Engineering, Technomic, Lancaster, UK, 1992.

[18] E. Yilmaz, N. Erdenizci, and O. Yilmaz, "Miscibility of chitosan and poly(ethylene oxide) in dilute Solution," International Journal of Polymer Analysis and Characterization, vol. 8, no. 5, pp. 327-338, 2003.

[19] P. A. Albertson, Partition of Cell Particles and Macromolecules, Wiley, New York, NY, USA, 3rd edition, 1986.

[20] P. A. A. P. Mamza and F. M. Folaranmi, "Compatibility studies on solution of polystyrene and polyvinyl acetate blend by density and viscometric methods," European Polymer Journal, vol. 32, no. 7, pp. 909-912, 1996.

[21] M. Mucha and A. Pawlak, "Thermal analysis of chitosan and its blends," Thermochimica Acta, vol. 427, no. 1-2, pp. 69-76, 2005.

[22] C. Bonnet-Gonnet, S. Leikin, S. Chi, D. C. Rau, and V. Adrian Parsegian, "Measurement of forces between hydroxypropylcellulose polymers: temperature favored assembly and salt exclusion," Journal of Physical Chemistry B, vol. 105, no. 9, pp. 1877-1886, 2001.

[23] H. M. P. Naveen Kumar, M. N. Prabhakar, C. Venkata Prasad et al., "Compatibility studies of chitosan/PVA blend in $2 \%$ aqueous acetic acid solution at $30^{\circ} \mathrm{C}$," Carbohydrate Polymers, vol. 82, no. 2, pp. 251-255, 2010.

[24] S. R. Illiger, C. Fadnis, T. Demappa, J. Jayaraju, and J. Keshavayya, "Miscibility studies of HPMC/PEG blends in water by viscosity, density, refractive index and ultrasonic velocity method," Carbohydrate Polymers, vol. 75, no. 3, pp. 484-488, 2009.

[25] K. Prasnt. Vasanthakumari, S. K. Rai, and H. Shiringara, "Studies on the mechanical properties of unsaturated polyester toughened epoxy/silk composites," International Journal of Polymer Materials, vol. 25, no. 1, pp. 33-41, 2006.

[26] S. D. Ravi Prakash and S. K. Rai, "Miscibility studies on sodium alginate/polyethylene glycol blend in solution by refractive index, ultrasonic velocity, density and viscometric 
methods," International Journal of Plastics Technology, vol. 8, p. 334, 2004.

[27] W. Schenk, D. Reichert, and H. Schneider, "Compatibility in polymer blends of poly(vinyl acetate) and poly(methyl methacrylate) studied by nuclear magnetic relaxation," Polymer, vol. 31, no. 2, pp. 329-335, 1990.

[28] R. Mikkonen and A. Savolainen, "Blends of low density polyethylene/plasticized poly(vinyl chloride). The rheological, morphological, and mechanical characterization," Journal of Applied Polymer Science, vol. 39, no. 8, pp. 1709-1725, 1990.

[29] M. A. Sidkey, A. M. A. El Fattah, A. A. Yehia, and N. S. A. El All, "Ultrasonic investigation of some rubber blends," Journal of Applied Polymer Science, vol. 43, no. 8, pp. 1441-1449, 1991.

[30] K. C. Basavaraju, T. Demappa, and S. K. Rai, "Miscibility studies of polysaccharide Xanthan gum and PEO (polyethylene oxide) in dilute solution," Carbohydrate Polymers, vol. 69, no. 3, pp. 462-466, 2007.

[31] G. W. Adams and J. M. G. Cowie, "Blends of rigid and flexible macromolecules: poly(benzimidazole) mixed with poly(vinyl acetate-star-vinyl alcohol) copolymers," Polymer, vol. 40, no. 8, pp. 1993-2001, 1999.

[32] M. Mathew, K. N. Ninan, and S. Thomas, "Compatibility studies of polymer-polymer systems by viscometric techniques: nitrile-rubber-based polymer blends," Polymer, vol. 39, no. 25, pp. 6235-6241, 1998.

[33] R. K. Wanchoo and P. K. Sharma, "Viscometric study on the compatibility of some water-soluble polymer-polymer mixtures," European Polymer Journal, vol. 39, no. 7, pp. 14811490, 2003.

[34] T. Cai, Z. B. Hu, B. Ponder, J. St. John, and D. Moro, "Synthesis and study of and controlled release from nanoparticles and their networks based on functionalized hydroxypropylcellulose," Macromolecules, vol. 36, no. 17, pp. 6559-6564, 2003. 


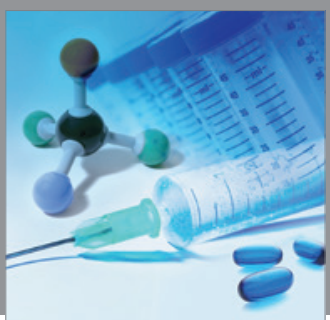

International Journal of

Medicinal Chemistry

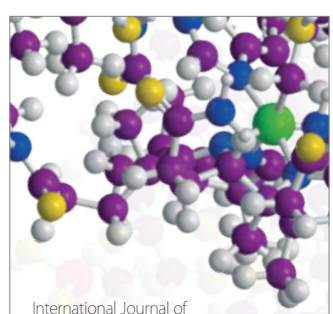

Carbohydrate Chemistry

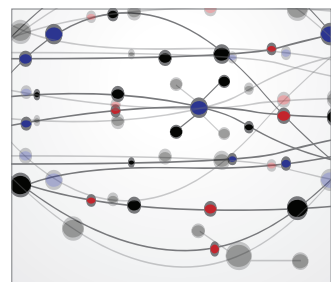

The Scientific World Journal
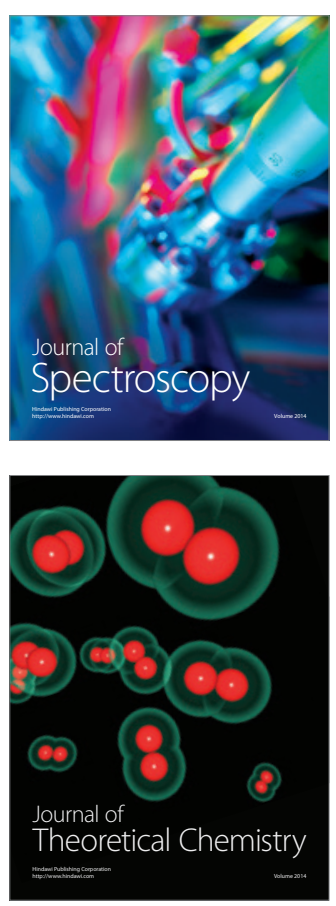
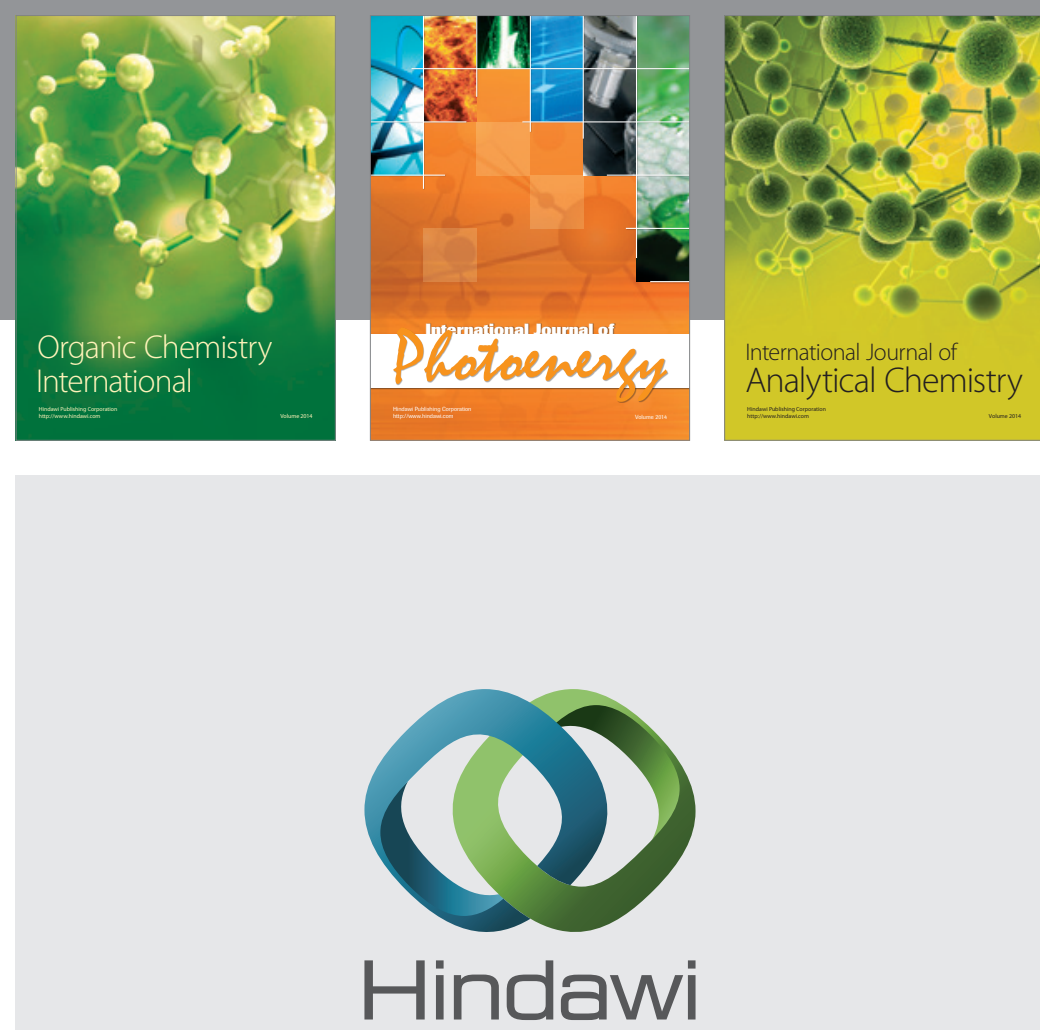

Submit your manuscripts at

http://www.hindawi.com
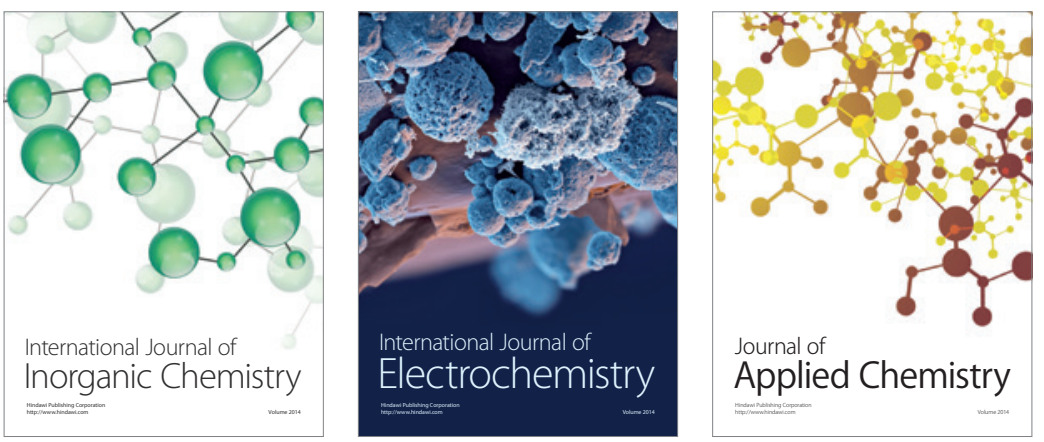

Journal of

Applied Chemistry
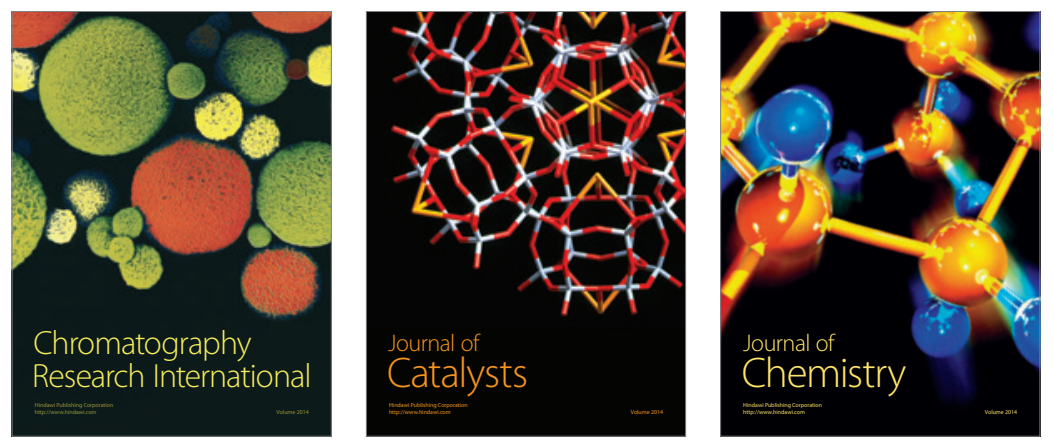
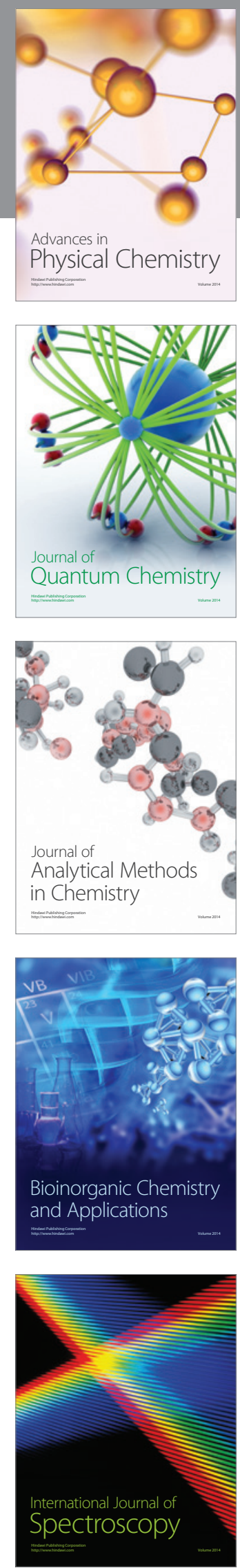